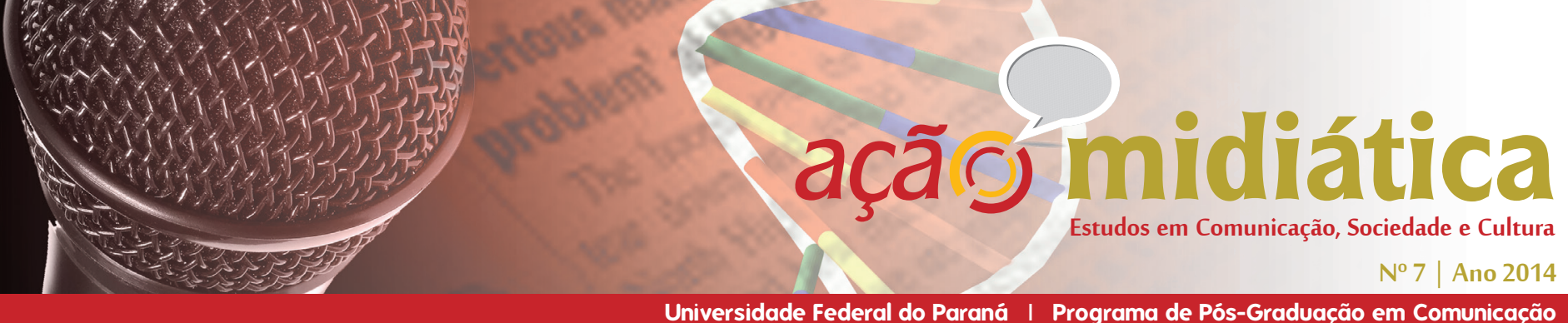

\title{
O Estado da Arte da Pesquisa em Divulgação Científica no Brasil: Apontamentos Iniciais ${ }^{1}$
}

\author{
The Current State of Research on Science Writing and Popular Science in Brazil: Initial \\ Findings
}

El Estado del Arte de la Investigación de Divulgación Cientifica en Brasil: Notas Preliminares

\author{
Graça CALDAS ${ }^{2}$ \\ Kátia ZANVETTOR ${ }^{3}$
}

\begin{abstract}
Resumo
Este artigo traz resultados iniciais do Projeto "Divulgação Científica e Sociedade: Memória e Estado da Arte", do Grupo de Pesquisa "Comunicação, Educação, Ciência e Sociedade", do Labjor/Unicamp. Elabora um diagnóstico nacional sobre a pesquisa em Divulgação Científica, nos programas de Pós-Graduação do país. Inicialmente foi realizado um mapeamento no banco de teses da Capes, a partir de palavras-chave relacionadas à área, com destaque para a produção do Estado de São Paulo. O método de abordagem é descritivo e experimental, de natureza quantitativa e qualitativa. Os primeiros resultados da análise de seis palavras-chave computam o registro de 761 trabalhos, em todo o Brasil. Restringindo a análise para três palavras-chave aparecem 338 trabalhos, sendo que 78 ocorrem nas universidades do Estado de São Paulo. Os destaques são para "Divulgação Científica", com maior incidência na Unicamp (27), seguida da USP (26), UMESP (11) e UFSCAR (8). Os resultados indicam, ainda, que os trabalhos são de natureza múltipla e integram grupos de pesquisa de diferentes áreas do conhecimento.
\end{abstract}

Palavras-chave: Divulgação científica; Estado da arte; Pesquisa; Brasil; São Paulo.

\section{Abstract}

This text presents initial results of the project "Science Writing and Society: Memoirs and State of the Art" developed by the Research Group on "Communication, Education, Science and Society" of the Labjor/Unicamp (Journalism Lab, University of Campinas). The project intends to produce a countrywide assessment of research on Science Writing in the graduate programs in Brazil. The starting point was a survey in the public archive of theses (Ph.Ds

1 Trabalho apresentado à sétima edição da Revista Ação Midiática - Estudos em Comunicação, Sociedade e Cultura, publicação ligada ao Programa de Pós-Graduação em Comunicação, da Universidade Federal do Paraná.

2 Graça Caldas é jornalista, Doutora em Ciências da Comunicação (ECA/USP), Pós-Doutorado em Política Científica no DPCT/IG/Unicamp. Docente do Programa de Pós-Graduação em Divulgação Científica e Cultural do Labjor/IEL/Unicamp. Líder do Grupo de Pesquisa do CNPq no Labjor/Unicamp, em "Comunicação, Educação, Ciência e Sociedade". Coordenadora da pesquisa "Divulgação Científica e Sociedade: Memória e Estado da Arte", Bolsista Produtividade do CNPq em Divulgação Científica. E-mail: gcaldas@unicamp.br

3 Kátia Zanvettor é jornalista, Doutora em Educação (USP). Professora de Comunicação na Universidade do Vale do Paraíba (Univap). Pesquisadora colaboradora no Labjor/Unicamp onde desenvolve a pesquisa "Divulgação Científica e pesquisa: a contribuição do Estado de São Paulo" como projeto de Pós-Doutorado. Integra o Grupo de Pesquisa do CNPq no Labjor/ Unicamp, em "Comunicação, Educação, Ciência e Sociedade". Líder do Laboratório de Pesquisa e Estudo em Comunicação e Cultura Labcom/Univap. E-mail: katia.zanvettor@gmail.com 


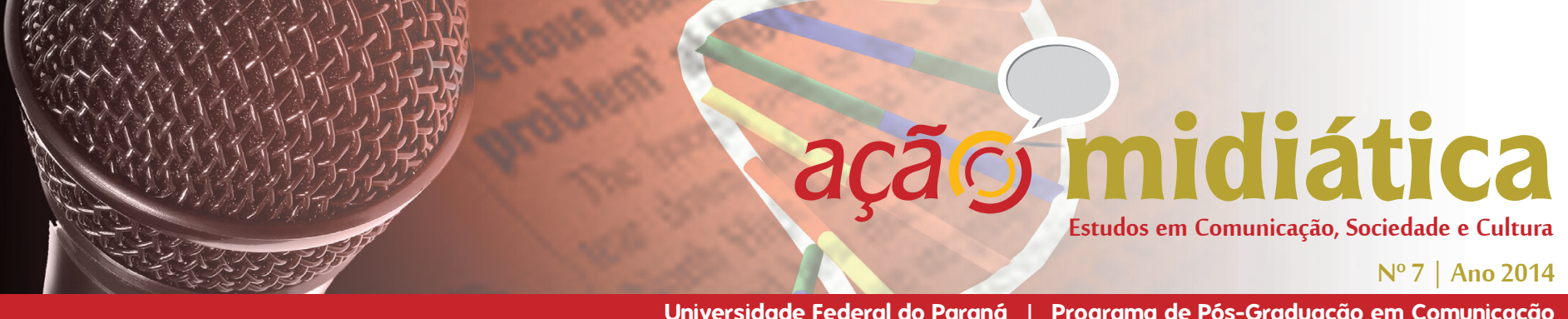

and Masters) maintained by CAPES (the Brazilian graduate program funding agency), using specific keywords for this field of knowledge, with emphasis on the State of São Paulo. Our approach is descriptive and experimental, both qualitative and quantitative. The first listing, based on six keywords, encompassed 761 theses presented across Brazil. Restricting the search to three main keywords narrowed the outcome to 338 theses, of which 78 were presented in universities of the State of São Paulo. The foremost keyword was "Divulgação Científica" (Science Writing, or Popular Science) which retrieved 27 theses produced in the University of Campinas (Unicamp), followed by the University of São Paulo (USP, 26), the Methodist University of São Paulo (UMESP, 11) and the Federal University of São Carlos (8). The studies presented in these theses are of a multiple nature and they were produced within research groups of different fields of knowledge.

Keywords: Science writing; State of art; Research; Brazil; São Paulo.

\section{Resumen}

Este artículo presenta los primeros resultados del proyecto "Divulgación de la Ciencia y Sociedad: Memoria y Estado del Arte", del Grupo de Investigación "Comunicación, Educación, Ciencia y Sociedad", del Labjor/Unicamp. Elabora un diagnóstico nacional sobre la investigación en Divulgación Científica en los programas de posgrado en Brasil. Como punto de partida se realizó una busqueda en las bases de datos da Capes-Brasil a partir de las palabras-clave relacionadas con la área, con un recorte en la producción del Estado de São Paulo (Brasil). El método de acercamiento es descriptivo y experimental, cuantitativo y cualitativo. Los primeros resultados de la análisis de seis de las palabras-clave destacadas apuntan 761 trabajos, en todo el Brasil. Restringiendo el análisis a tres palabras-clave aparecen 338 trabajos, de los cuales 78 ocurren en las universidades del Estado de São Paulo. Los aspectos mas destacados son para "Divulgación Científica", centrándose en la Unicamp (27), seguido por la USP (26), UMESP (11) y UFSCAR (8). Los resultados también indican que los trabajos son de naturaleza múltiple y integran grupos de investigación de diferentes áreas del conocimiento.

Palabras clave: Divulgación científica; Estado del arte; Investigación; Brasil; São Paulo.

\section{Introdução}

Nos últimos anos o Brasil tem ampliado, substancialmente, sua produção científica. Os dados nacionais são reflexo do trabalho de 129 mil pesquisadores ativos em 28 mil grupos de pesquisa credenciados pelo $\mathrm{CNPq}$, em 452 instituições. Os indicadores de produção nacional em CT\&I realizados pela Fapesp em 2010 (FAPESP, 2011) mostram que a contribuição nacional para a produção científica mundial passou de 1,6\% em 2002 (13.180) para 1,9\% (18.915) em 2006, na aferição realizada nas bases SCIE (Science Citation Index Expanded) e SSCI (Social Science Citation Index). Nesse mesmo período, foi possível observar que a taxa de crescimento das publicações do Brasil foi praticamente o dobro do crescimento mundial. Enquanto o Brasil alcançou um índice de 43,5\% de crescimento 
nesses quatro anos, o restante do mundo cresceu $22,7 \%{ }^{4}$

Dados mais recentes divulgados por Righetti (2013) a partir dos dados do ranking da SCIimago Journal Rank (SJR), com base nos dados Scopus, indicam que o Brasil, em uma década, de 2001 a 2011, subiu quatro degraus na posição mundial, saindo do $17 .^{\circ}$ lugar para o 13. ${ }^{\circ}$. Essa mudança de patamar foi possível porque o número de papers brasileiros publicados no período saltou de 13.846 para 49.664. O ranking computa os resultados de 238 países.

Embora os indicadores nacionais e internacionais apontem para um crescimento relevante da produção acadêmica brasileira na área de Ciência, Tecnologia e Inovação (CT\&I), o aproveitamento dessa produção para o desenvolvimento econômico, social e tecnológico do país ainda é considerado baixo. Além disso, há um descompasso entre a produção científica e sua divulgação para a sociedade. Quais seriam as causas deste gap? Como a comunicação pública da ciência em diversos formatos e plataformas para diferentes públicos pode contribuir para a democratização do conhecimento? Quais são os temas que compõem a agenda de pesquisa na área de Divulgação Científica (DC)? Em que áreas de conhecimento estão mais presentes e que apoios recebem das agências de fomento?

Questões como essas fazem parte do projeto de pesquisa "Divulgação Científica e Sociedade: Memória e Estado da Arte", do Grupo de Pesquisa "Comunicação, Educação, Ciência e Sociedade", do Labjor/Unicamp, liderado pela pesquisadora Graça Caldas e integrado pela pesquisadora Kátia Zanvettor. O projeto pretende desenvolver um diagnóstico nacional sobre a pesquisa da área, suas instituições, seus temas mais pesquisados e metodologias empregadas.

\section{Discurso e práxis}

Em novembro de 2013, durante a realização da sexta edição do Fórum Mundial da Ciência, realizado no Rio de Janeiro, cientistas de vários países, incluído os presidentes da SBPC, Helena Nader, e da Academia Brasileira de Ciências, Jacob Palis, ratificaram a importância da divulgação. No documento final do fórum, entre os cinco tópicos destacados, a área de Educação e a Difusão da Ciência estiveram presentes, demonstrando a centralidade do debate sobre a divulgação e o reconhecimento nacional e internacional para o relevante papel da comunicação pública da CT\&I.

Porém, da mesma forma que existe um descompasso entre a produção científica nacional e sua divulgação para a sociedade, observa-se uma falta de sintonia entre o discurso dos cientistas, dos governantes e das agências de fomento com relação ao apoio às pesquisas da

4 Segundo o relatório da Fapesp (2010) os valores absolutos de artigos publicados no Mundo entre 2002 e 2006 são de 4.616.780 artigos publicados. O Brasil publicou 79.619 artigos indexados no mesmo período. 


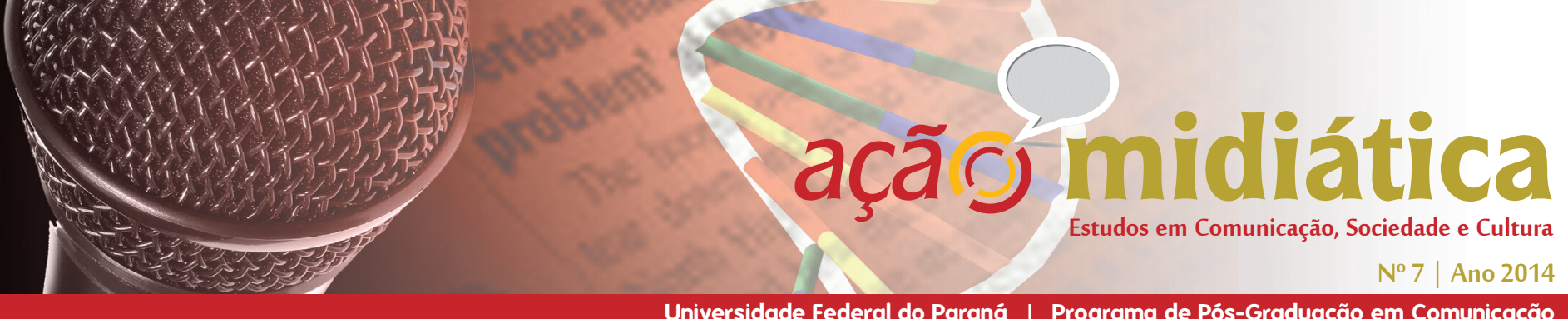

área de Divulgação Científica. Só recentemente (2012), o CNPq criou uma área de Divulgação Científica para o encaminhamento de projetos de pesquisa na área, com bolsas de pós-graduação, bem como bolsa produtividade, até então restrito a outros setores. Além disso, abriu uma aba na Plataforma Lattes para a inserção de produção técnica em Divulgação Científica não apenas para jornalistas-pesquisadores, mas para cientistas em geral (reportagens, entrevistas, artigos de divulgação, entre outras possibilidades).

Ainda que todas essas iniciativas representem um avanço importante, os editais nas diferentes agências de fomento ainda são insuficientes face à alta demanda do setor. Paulatinamente, porém, as Fundações de Apoio à Pesquisa do País vêm ampliando os editais específicos, bem como criando Prêmios de Jornalismo Científico para estimular as notícias na mídia em geral. A Fapesp, por sua vez, conta com um uma linha especial de recursos para a atuação de jornalistas, o Mídia Ciência.

Por outro lado, apesar do discurso favorável à produção de pesquisas em projetos de pesquisa em Divulgação Científica, há, ainda, poucas ações efetivas para garantir o real compartilhamento do conhecimento científico com toda a sociedade (ROQUEPLO, 1974).

Há uma falta de reconhecimento da importância da ciência para o desenvolvimento do próprio país, em grande parte devido à carência da disseminação de conhecimentos sobre a ciência nacional nos órgãos de comunicação, agências governamentais, empresas, sociedade em geral e mesmo na própria comunidade científica. Essa falta de informação pode acarretar também o uso inadequado de informações e procedimentos de análise da produção científica, bem como sua perda de credibilidade (FAPESP, 2011, v. 1, p. 7).

Somente por meio de pesquisas sobre Divulgação Científica e da ampla divulgação da produção científica, seus riscos e benefícios, a sociedade brasileira poderá, efetivamente, participar do debate nacional e posicionar-se em temas polêmicos que afetem o interesse público. Assim, conseguirá entender melhor o papel da CT\&I no desenvolvimento político, econômico e social do país.

Como explica Caldas (2010), nesse processo é preciso observar não apenas aspectos técnicos, mas que envolvem também princípios éticos e políticos, que contribuam para uma visão crítica da CT\&I pela sociedade.

Considerando que quase tudo que acontece na sociedade é influenciado pela C\&T, é preciso que o discurso científico seja amplamente compreendido pela população, para que possa tomar suas decisões a partir de múltiplas informações, considerando os aspectos positivos e negativos de cada situação. Não se trata, obviamente, de negar a especificidade dos saberes, nem de abrir mão deles, mas, sim, de possibilitar a participação efetiva da sociedade em debates públicos sobre temas polêmicos, como transgênicos, biotecnologia, energia nuclear, entre tantos outros, cujos impactos sociais são inegáveis (CALDAS, 2010, p.33). 
Por outro lado, é importante lembrar que a ciência, a tecnologia e a inovação são campos de produção do saber que estão articulados com diferentes formas de poder, no sentido proposto por Foucault (1979), não homogêneo ou exclusivamente aparelhado às instituições que sustentam as sociedades desiguais. Suas produções têm um impacto significativo na vida das pessoas. Por essa razão, possibilitar o acesso não só dos resultados da produção científica, mas também de seu processo e de toda a cadeia produtiva, discutindo eventuais interesses envolvidos, legítimos ou não, é uma forma de tornar transparentes essas redes de poder, incluindo suas contradições.

Logo, a comunicação pública da ciência e da tecnologia deve ser precisa e articulada historicamente, incluindo as controvérsias naturais do processo de produção e das relações de poder entre seus atores sociais. Mesmo porque, ela não é uma mera técnica ou pretensamente técnica (BUENO, 2013). A atividade de divulgação da ciência deve permitir a reflexão, a crítica e o acesso ao "contraditório" (CALDAS, 2010, p. 32). Essa possibilidade reflexiva é fundamental para a formação de uma cultura científica cidadã da sociedade.

Com base nas perspectivas levantadas acima sobre o papel da Comunicação na área de Divulgação Científica e de sua difusão, observa-se que para que esta tenha efetivada sua propagada contribuição social, deve se materializar em uma perspectiva qualificada e crítica. Trata-se, portanto, não só de divulgar a produção da ciência, mas de possibilitar a apreensão desse conhecimento, para que o público em geral possa não apenas compreender seus processos, mas também participar de decisões sobre temas de interesse da sociedade.

\section{Objetivos e metodologia}

O projeto tem por objetivo fazer um diagnóstico nacional sobre o estado da arte do conhecimento em Divulgação Científica (DC), no Brasil. Pretende desenvolver indicadores quantitativos e qualitativos da área. Examinará as linhas de pesquisas em DC e suas interfaces, seus pesquisadores e sua produção acadêmica nos programas de pós-graduação em Comunicação e nas demais áreas do conhecimento. Fará um mapeamento das dissertações e das teses, observando os temas abordados, as instituições, bem como as metodologias e os referenciais teóricos utilizados. Nos cursos de graduação em Comunicação identificará as disciplinas de Jornalismo Científico, seus professores, as obras de referência utilizadas, bem como os trabalhos de conclusão de curso (TCCs) em DC.

O método de abordagem é descritivo e experimental, de natureza qualitativa e quantitativa. Utiliza a pesquisa bibliográfica e documental. Na primeira etapa da pesquisa realizada, em maio de 2014, foi produzido um mapeamento geral das pesquisas em Divulgação Científica no Banco de Teses da Capes, a partir de seis palavras-chave: Divulgação Científica, Jornalismo Científico, Popularização da Ciência, Comunicação Pública da Ciência, Comunicação 


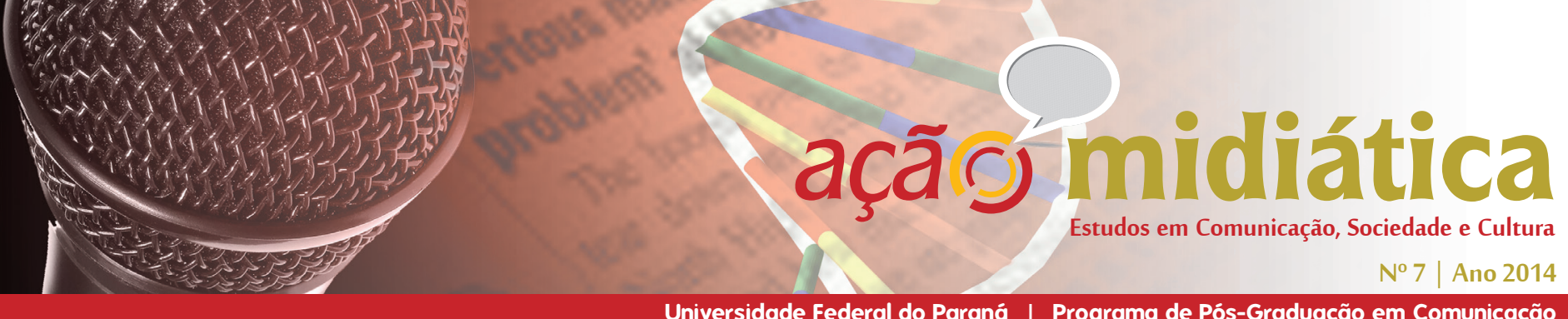

e Educação, e Comunicação, Tecnologia, Ciência e Sociedade para verificar as ocorrências de trabalhos na área.

A ideia é identificar e examinar as principais interfaces de pesquisas em Divulgação Científica realizadas na área, em todo o Brasil. Inicialmente fizemos um recorte, no Estado de São Paulo, com as três primeiras palavras-chave. Em seguida examinamos as pesquisas desenvolvidas no Mestrado Multidisciplinar de Divulgação Científica e Cultural do Labjor/IEL/ Unicamp, referência na área e com 48 dissertações defendidas em quatro anos (2010-2014). Os resultados preliminares dessa fase da pesquisa são apresentados neste artigo.

\section{Resultados da pesquisa em DC}

Nos 45 programas da área de Comunicação do país, 23 estão localizados na região Sudeste; nove no Sul; sete no Nordeste; quatro no Centro-Oeste e dois no Norte. Nesses programas, ao contrário do que se poderia esperar, nenhuma das áreas de concentração refere-se especificamente à Divulgação Científica. O nome não aparece nem mesmo em suas linhas de pesquisa.

As áreas de concentração desses programas estão assim constituídas: Ciências da Comunicação (03), Comunicação Social (03), Comunicação (21), Comunicação e Cultura (01), Comunicação e Cultura Contemporânea (01), Comunicação e Culturas Midiáticas (01), Comunicação e Informação (01), Comunicação e Linguagens (01), Comunicação e Práticas de Consumo (01), Comunicação e Semiótica (01), Comunicação e Territorialidades (01), Comunicação Social: Ingerações Midiáticas (01), Comunicação Cultura e Amazônia (01), Estudos da Mídia (01), Imagem e Som (01), Jornalismo (03) Meios e Processos Audiovisuais (01), Mídia e Cotidiano (01) e Multimeios (01).

A única linha de pesquisa dos programas de pós em Comunicação que explicitava a área, a de Comunicação Científica e Tecnológica da Universidade Metodista de São Paulo, pioneira, deixou de existir em 2010, depois de uma vasta produção na área, orientada pelos pesquisadores Isaac Epstein, Wilson da Costa Bueno, Graça Caldas e Elizabeth Gonçalves. Por outro lado, observa-se que a pesquisa em Divulgação Científica está pulverizada em grupos de pesquisa não só da área de Comunicação, mas de muitas outras, com destaque para o setor de Educação, Linguagem, Geociências e História da Ciência. Cursos específicos como os de Ciência, Tecnologia e Sociedade (CTS) e o Mestrado Multidisciplinar da Unicamp em Divulgação Científica e Cultural (MDCC) apontam a tendência do setor de se constituir como um campo multidisciplinar.

Como parte inicial desse diagnóstico para identificar as pesquisas e as instituições em todo o Brasil, foi realizado um levantamento geral no Banco de Teses e Dissertações da Capes, maio de 2014, tendo como referência seis palavras-chave relacionadas ao tema do estudo. São 
elas: Divulgação Científica; Jornalismo Científico; Popularização da Ciência; Comunicação Pública da Ciência; Comunicação, Educação e Ciência e Comunicação, Tecnologia, Ciência e Sociedade. Os primeiros resultados computam o registro de 761 trabalhos.

Nesse primeiro levantamento, o número de ocorrências foi assim distribuído, de forma decrescente: Divulgação Científica (215); Educação e Ciência (201); Comunicação Pública da Ciência (114); Comunicação, Tecnologia, Ciência e Sociedade (108); Popularização da Ciência (97) e Jornalismo Científico (26). Com base nesses resultados é possível inferir e ratificar as primeiras impressões colhidas no mapeamento dos grupos de pesquisa do CNPq, onde essas pesquisas estão localizadas. A agenda prevalente da Divulgação Científica nas pesquisas brasileiras permeia o interesse de pesquisadores de diferentes campos do conhecimento, por problemáticas que relacionam Ciência e Sociedade, em uma perspectiva multidisciplinar e não apenas da Comunicação, como seria possível imaginar. O fato de o Jornalismo Científico ter obtido apenas 26 ocorrências é também revelador.

Após esse levantamento das seis palavras-chave em pesquisas desenvolvidas em todo o território nacional, filtrou-se essa produção em uma segunda etapa do trabalho para o refinamento de catalogação das publicações, tomando como recorte os trabalhos produzidos nas universidades do Estado de São Paulo, considerando a relevância de sua produção científica em relação ao resto do país. Dessa vez, porém, o levantamento foi restrito a apenas três palavras-chave que receberam, respectivamente, as seguintes ocorrências: Divulgação Científica (53), Jornalismo Científico (09) e Popularização da Ciência (16), totalizado 78 trabalhos em São Paulo com essas palavras-chave.

O maior número de ocorrências de trabalhos com a palavra-chave "Divulgação Científica" no Estado de São Paulo foi na Unicamp (27), seguida da USP (26), da Universidade Metodista de São Paulo (11) e da Universidade Federal de São Carlos (8). Ainda no Estado de São Paulo, para "Jornalismo Científico" foram localizados nove, sendo três na Unicamp, dois na PUC-SP, dois na Umesp, um na Unesp e um na Unip. Por fim, "Popularização da Ciência" teve 16 ocorrências. As universidades com maior número de registros nessa palavra foram a USP, com seis trabalhos, a PUC-SP, com três e a Unicamp, com dois.

Assim, observamos que no Estado de São Paulo as instituições que, de modo geral, computando as três palavras-chave analisadas, mais publicaram foram a Unicamp e a USP, com 32 trabalhos cada uma, a Umesp, com 13, e UFSCAR com oito trabalhos.

\section{Labjor-Unicamp: pesquisas e temas}

O Laboratório de Estudos Avançados em Jornalismo (Labjor), segundo a definição institucional disponível em sua webpage, http://www.labjor.unicamp.br, "é um centro de referência, no país e na América Latina, para a formação e para os estudos em divulgação 


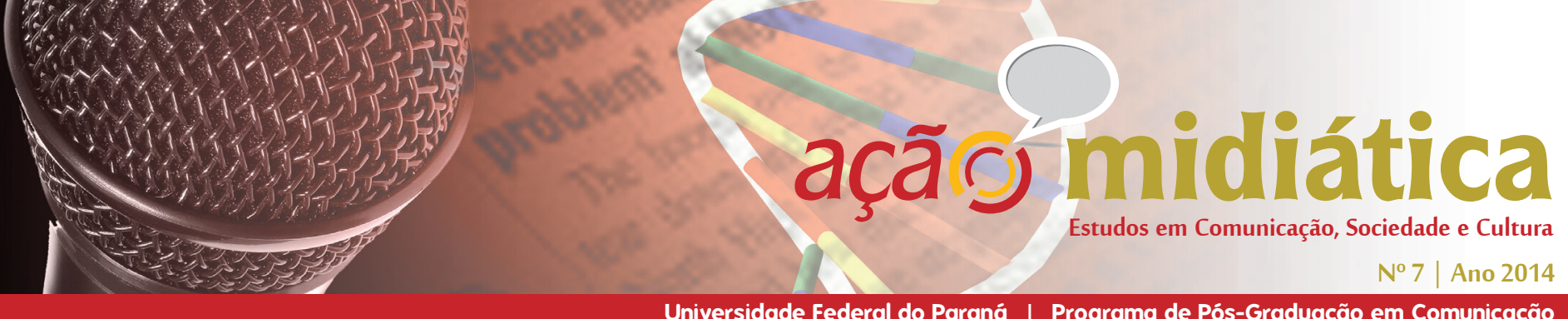

As 48 dissertações defendidas no Programa do Labjor/IEL/Unicamp foram orientadas por 20 professores que são, em sua maioria, professores plenos, mas também há a colaboração de outros professores participantes. Em termos de quantidade de orientações, percebe-se uma distinção na distribuição e atuação dos orientadores. Os professores com maior número de trabalhos orientados desde o início do mestrado no programa foram, na sequência, Graça Caldas (10), Cristiane Pereira Dias (05), Vera Regina Toledo Camargo (4), Susana Oliveira Dias (4), Rafael de Almeida Evangelista (3) e Antonio Carlos Rodrigues de Amorim (3). Os demais professores aparecem com dois ou um trabalho.

\section{Temas prevalentes}

Após o levantamento das dissertações defendidas no Labjor, com a organização dos respectivos alunos e orientadores, passamos para uma etapa de análise temática, partindo, nesse primeiro momento, única e exclusivamente, dos títulos das dissertações. Assim, a categoria proposta aqui é uma aproximação. Em etapa posterior serão examinados seus resumos e metodologias para maior detalhamento. Assim, chegamos a oito categorias de temas prevalentes: Política Científica; Linguagem; Ciência; Ciência da Saúde; Assessoria de Imprensa; Ciência do Esporte; Educação e Outros. Na categoria “Outros” foram colocados aqueles trabalhos que não permitiram fazer nenhuma inferência ou aproximação temática apenas pelo título.

É importante a observação de que, tratando-se de um programa de Divulgação Científica e Cultural, entende-se que, de um modo ou de outro, todos os trabalhos discutem ou se aproximam da questão da ciência. Contudo, consideramos necessária uma categoria denominada "Ciência" porque alguns trabalhos, notadamente, dão ênfase maior a esse objeto em seu título que outros.

Logo, as categorias com maior ocorrência são Ciência, com 14 trabalhos, Linguagem, com 10, e Ciências da Saúde, com sete. Em seguida vem Educação, com seis trabalhos, Outros, com cinco, Ciência do Esporte, com três, Política Científica, com dois, e Assessoria de Imprensa, com um trabalho, totalizando os 48 trabalhos analisados.

\section{Considerações finais}

Os resultados preliminares deste trabalho apontam que o campo de estudos da Divulgação Científica encontra-se em expansão e que atravessa não só a área de Comunicação como tem afinidade temática com outras áreas, revelando ao mesmo tempo sua complexidade e sua natureza multidisciplinar. Considerando que a divulgação científica nos meios de comunicação tem sido pautada, majoritariamente, pelos resultados da pesquisa, com problemas de precisão de informações e de dificuldades de entendimento do público em geral, mapear o 
